\title{
Arqueología de la Arquitectura, una mirada desde América del Sur
}

\section{Archaeology of Architecture, a view from South America}

\author{
Andrés Zarankin ${ }^{1}$ \\ Departamento de Antropologia y Arqueologia, UFMG, Brasil \\ Pedro Paulo A. Funari² \\ Departamento de História, Unicamp, Brasil
}

\section{RESUMEN}

La Arqueología de la Arquitectura en América del Sur, a pesar de diversa y heterogénea, posee algunas características distintivas. Una de estas ha sido un creciente interés por discutir los sistemas de reproducción de las estructuras de poder en la región. Concibiendo la arquitectura como una tecnología del poder y una forma de comunicación no verbal, en este trabajo sintetizamos propuestas teórico metodológicas y casos de estudio, que hemos desarrollado a lo largo de 20 años.

Palabras clave: América del Sur; Arqueología; casas; escuelas; centros clandestinos de detención; muros.

\begin{abstract}
The Archeology of Architecture in South America, despite being diverse and heterogeneous, owns some distinctive characteristics. One of these has been a growing interest in discussing the strategies for reproducing power systems in the region. Understanding architecture as a technology of power and a non-verbal type of communication, in this paper we present a short synthesis of theoretical methodological proposals and study cases, which we have worked over the last 20 years.
\end{abstract}

Keywords: South America; Archaeology; houses; schools; clandestine detention centers; walls.

Recibido: 27-09-2019. Aceptado: 04-05-2020. Published online: 16-12-2020

Citation / Cómo citar este artículo

Zarankin, A. y Funari, P. P. A. 2020: "Arqueología de la Arquitectura, una mirada desde América del Sur", Arqueología de la Arquitectura, 17: e103. https://doi.org/10.3989/arq.arqt.2020.011

Copyright: (c) CSIC, 2020. (C) UPV/EHU Press, 2020. Este es un artículo de acceso abierto distribuido bajo los términos de la licencia de uso y distribución Creative Commons Reconocimiento 4.0 Internacional (CC BY 4.0).

\footnotetext{
zarankin@yahoo.com / ORCID iD: http://orcid.org/0000-0002-0020-0606

2 ppfunari@uol.com.br/ORCID iD: http://orcid.org/0000-0003-0183-7622
} 


\section{INTRODUCIÓN}

Uno de los elementos distintivos de la especie humana es su capacidad de proyectar, diseñar y construir su propio hábitat. El resultado de este proceso es una heterogeneidad de estructuras materiales distribuidas en el paisaje a las que denominamos "Arquitectura". Desde sus inicios la Arqueología, se ha interesado en estudiar y analizar la Arquitectura de grupos en el pasado, apenas como una más entre las tantas evidencias materiales existentes. Sin embargo es únicamente a partir de finales del siglo XX que se consolida a nivel mundial un campo de investigación específico dentro de la Arqueología denominado "arqueología de la arquitectura"3 (Steadman 1996, 2015; Azkarate 2001, 2013; Mañana Borrazás, Blanco Rotea y Ayán Vila 2002; Ayán 2003). En América del Sur, este tipo de estudios vienen creciendo y diversificándose y es posible encontrar trabajos de análisis arquitectónicos/espaciales en sitios prehistóricos Nielsen (1995), fuertes coloniales (Soares 2015), instituciones totales contemporáneas (Brandão 2018) o casas actuales (Zarankin 1999). Al mismo tiempo, los enfoques teóricos utilizados en estos trabajos son bastante amplios. Personalmente nos interesa utilizar la arqueología de la arquitectura para discutir los sistemas de reproducción de las estructuras de poder a través de las prácticas cotidianas (Zarankin y Senatore 2002; Funari y Zarankin 2004).

Desde una arqueología del pasado cercano/reciente/ contemporáneo/presente (Buchli y Lucas 2002; Ruibal 2014) nos proponemos traer una discusión que expone situaciones de reproducción de desigualdad y violencia social, y por lo tanto ayuda a construir una sociedad más democrática y justa (Funari 1997). Como parte de esa búsqueda ética por una práctica arqueológica libertaria, en este artículo recuperamos algunas cuestiones que ya presentamos en el pasado para retomar la discusión sobre el lugar que puede tener la Arqueología en el estudio del universo cotidiano moderno, en particular la Arqueología de la Arquitectura. Utilizaremos para nuestro análisis algunos casos de estudio sobre casas familiares y escuelas primarias, a partir de la evidencia

\footnotetext{
"Arqueología de la arquitectura" es un campo amplio de estudio que incluye una gran diversidad de temas y enfoques teóricos, centrados en el análisis arqueológico de estructuras constructivas. Este tipo de abordajes sobre la materialidad de la arquitectura, ha demostrado un alto potencial para ayudar a comprender diversos aspectos relacionados con la conformación del paisaje humano.
}

porteña ${ }^{4}$, a los cuales incluiremos análisis de los campos de detención en contexto dictatorial, en Argentina y muros perimetrales en casas de la ciudad de Belo Horizonte en Brasil.

\section{ARQUEOLOGÍA DE LO COTIDIANO}

Lo "cotidiano" es aquello que está presente en el día a día de las personas. Un espacio en el cual el sistema de poder utiliza sus estrategias de reproducción y disciplina más productivos. Es en el cotidiano y en sus prácticas que se construye la sociedad. Alimentarse, vestirse, habitar el espacio, son todos elementos de este cotidiano que pueden ser fuente del trabajo de la Arqueología. Es por este motivo que varios de nuestros trabajos están relacionados con reflexiones de los mecanismos reproductivos de la sociedad capitalista a partir de una Arqueología de la Arquitectura de lo "cotidiano" en la sociedad moderna (Orser 1996: Johnson 1996), especialmente centrada en análisis de estructuras construidas, así como de la organización del espacio (Zarankin 1999, 2002, 2005a, 2008). Esta propuesta de alguna manera, tiene similitudes con lo que Daniel Miller (1987) llamó estudios de "cultura material", o estudios sobre la "invención de la vida cotidiana" por Michel De Certeau (1980).

Es notorio que existe un preconcepto con este tipo de estudios, ya que como señala Trigger (1989), para bastantes entre quienes se dedican a la arqueología, el trabajo que no involucra excavaciones o el estudio de objetos antiguos no puede llamarse "arqueológico". Personalmente, no compartimos esta posición ortodoxa, ya que consideramos que la diferencia de nuestro trabajo frente a especialistas en otras disciplinas es su capacidad para comprender e interpretar la "cultura material" (independientemente de variables como el espacio, el tiempo o la procedencia de los objetos analizados). Al mismo tiempo, una "arqueología del cotidiano" puede verse como una arqueología socialmente útil, ya que contribuye directamente a reflexionar sobre los principios ideológicos que estructuran nuestras vidas. Como señala Foucault (1976), solo entendiendo y exponiendo los dispositivos de reproducción del poder tendremos la posibilidad de generar cambios en la sociedad.

Un número importante de arqueólogas y arqueólogos de Sudamérica comparten esta visión y vienen

\footnotetext{
4 Natural de Buenos Aires.
} 
desarrollando investigaciones para debatir los rasgos que caracterizaron el día a día de la sociedad en contextos particulares (Funari 1995, 1999; Andrade Lima 1999; Orser y Funari 2001; Symanski 2001; Zarankin 2000, 2002; Zarankin y Senatore 2002; Salerno 2006; Gnecco y Ayala 2010; Haber 2011; Bezerra 2013; Pelllini 2016). De esta manera, la disciplina viene promoviendo una visión crítica y decolonial frente a los discursos que produjeron una comprensión generalizante, eurocéntrica y monolítica de la región.

\section{ARQUITECTURA COMO TECNOLOGÍA DEL PODER}

La explicación más frecuente sobre el origen y la función de la arquitectura está relacionada con la necesidad de protección/abrigo de los seres humanos (Zevi 1969; Nuttgens 1983; Conway y Roenish 1994). Quizás esta idea pueda justificar uno de sus principios básicos, pero evidentemente está lejos de explicar sus transformaciones y expansión a todos los aspectos de la existencia de las personas. De hecho, las ciencias sociales, desde múltiples perspectivas, se han interesado en discutir la naturaleza de la arquitectura y la organización del espacio (Panofsky 1957; Foucault 1976; King 1980; Gottdiener 1985, 1995; Lefevre 1991). Considerando que existe una enorme heterogeneidad de posiciones a este respecto, como mencionamos al inicio de este trabajo, nos interesan los enfoques que consideran la arquitectura como una herramienta que actúa como mediador entre una ideología (materializándola en una estructura y su organización espacial) y las personas (Hall 1966; Eco 1968; Grahame 1995, 1997).

Una vía para pensar la conexión entre ideología, Arquitectura y Arqueología es partir del principio de que el mundo se vuelve "acessible" a través de una serie de fijaciones en el espacio a las que llamamos lugar (Bachelard 1975). El espacio solo puede ser domesticado completamente, al transformarlo en "lugar", es decir, cuando es conocido, ocupado y utilizado. Generalmente esta trasformación se logra mediante dos estrategias principales; la imposición de un nombre a un determinado espacio; o a través de modificaciones en su materialidad, por lo que arquitectura se convierte en una herramienta central para domesticar el espacio. Al ser construcciones culturales, los lugares no son neutros. Como señala Foucault (1976) el arte de la distribución de personas y cosas en el espacio es una herramienta disciplinar del sistema de poder. La construcción del paisaje humano es el producto de la historia de la lucha por el poder, es decir, de enfrentamientos entre posiciones que intentan dominar y otras resistir. Controlando las políticas de construcción del paisaje, el sistema inventa dispositivos para autolegitimarse y reproducirse. La arquitectura es parte de esta manipulación al punto en que puede ser considerada una tecnología del poder (Foucault 1976; Grahame 1995).

Es justamente con el crecimiento de las ciudades dentro de un universo capitalista que son impuestas reglas constructivas generando así la popularización de una tendencia arquitectónica hacia una predominancia de la arquitectura académica controlada por el sistema. Estamos frente a un proceso de "arquitectonización" del paisaje y la vida humana, donde cada vez más actividades son realizadas dentro de estructuras construidas o dependen de ellas para poder ser llevadas a cabo. De la misma manera, la mayoría de las actividades y prácticas sociales cotidianas pasan a desarrollarse en el interior de edificios. En este contexto la arquitectura pasa a convertirse en un instrumento clave para la transmisión y reproducción del poder en el sistema capitalista (Sennett 1974; Markus 1993).

Esta intencionalidad (o ideología de la arquitectura), suele estar disimulada o escondida. Al respecto Foucault (1976), enfatiza que la arquitectura y su capacidad para distribuir, controlar y vigilar personas en el espacio es una estrategia del sistema para producir individuos disciplinados. La arquitectura se convierte así en una tecnología del poder.

\section{EL LENGUAJE DE LA ARQUITECTURA}

Toda acción humana es social; es decir, se asocia con el establecimiento de relaciones con otros (Bourdieu 1977; Giddens 1979, 1990). Del mismo modo, la cultura material, producto de esta acción, tiene la capacidad de transformarse en una herramienta para aproximarse a las personas, lo que de alguna forma representa el principio básico que sustenta la Arqueología (Hodder 1982; Shanks y Tilley 1987; Miller 1987). Entre diversos enfoques posibles, uno interesante es el hermenéutico o simbólico. Este parte de considerar la cultura material como un tipo de comunicación no verbal, por lo que su interpretación se convierte en uno de los grandes desafíos de la arqueología (Monks 1992; Fletcher 1989; Zarankin 1999, 2002). Esta comunicación no verbal ocurre mediante el uso de signos que carecen de una estructura 
sintáctica verbal, por lo que no se pueden analizar las secuencias de los componentes jerárquicos (Hall 1966).

Uno de los mecanismos para decodificar estos mensajes es la aplicación de modelos hermenéuticos. Como señalan Hodder y otros, para llevar a cabo esta "lectura" o análisis hermenéutico, es esencial lograr una reconstrucción contextual (Hodder 1994; Hodder et al. 1995). A partir de esta posición, podemos interpretar significados culturales dentro de contextos específicos. El acceso a contextos culturales en el pasado ha sido parte del desafío (y la crítica), de estos enfoques. Sin embargo, al aplicarlo a casos y situaciones de nuestra propia sociedad, esta tarea se ve facilitada.

Otra cuestión tiene que ver con las formas para "leer" discursos codificados en espacios y estructuras arquitectónicas (Glassie 1975; King 1980; Leone 1977; Gottdiener 1985; McGuire 1991; Kent 1990 Markus 1993; Parker Pearson y Richards 1994). Nos referimos a cuestiones que experimentamos a diario, de forma imperceptible, mientras nos movemos de un lugar a otro. Los circuitos, texturas, colores, luminosidades, amplitudes, tamaños, entre otros, son las variables a través de las cuales nuestro cuerpo decodifica estos discursos, generando diferentes sensaciones que rara vez traducimos a palabras de forma consciente (Knights 1994). A nivel académico, se generaron modelos para "traducir" el lenguaje no verbal, representado por el espacio y la arquitectura (Hage 1979; Hillier y Hanson 1984; Blanton 1994).

En nuestro caso consideramos extremadamente útil los resultados que surgen de la combinación entre el modelo "gamma" de Bill Hillier y Julienne Hanson (1984) y los índices propuestos por Richard Blanton (1994). Nos referimos a la posibilidad de acceder a un gráfico que explica la lógica espacial de la estructura arquitectónica, respaldada por resultados matemáticos que ayudan a entender variables como conectividad, distancia al exterior, grado de complejidad y tamaño de la construcción, entre otros.

Tomando como punto de partida nuestro interés por entender las estrategias reproductivas del sistema de poder, y utilizando la metodología de análisis de la arquitectura, a través del tiempo hemos efectuado diversos estudios tomando como casos de análisis elementos arquitectónicos de nuestro cotidiano como casas, escuelas, muros, instituciones bancarias, prisiones, entre otros (Funari y Zarankin 2002, 2005, 2006, 2010; Zarankin 1999, 2000, 2002, 2005a, 2005b, 2008, 2012; Zarankin y Niro 2006), algunos de los cuales iremos a presentar a continuación.

\section{CUERPOS CONGELADOS; CASAS Y MUROS PERIMETRALES}

Un primer caso de estudio que podemos definir como "experimental" sobre esta arqueología de la arquitectura de lo cotidiano, surge a partir de un trabajo de análisis de las paredes perimetrales de casas familiares en Brasil. Precisamente, una de las cosas que más llaman la atención de las ciudades brasileñas para quienes proceden del extranjero, son sus enormes muros, las omnipresentes rejas y los alambrados electrificados que la mayoría de las estructuras arquitectónicas exhiben como algo "natural y normal".

Como hemos discutido al inicio de este artículo, las justificativas más frecuentes relativas a la arquitectura tienen un carácter funcionalista simple (necesidad de refugio de las personas). También en este artículo venimos discutiendo otros abordajes alternativos para pensar el fenómeno de las prácticas constructivas y su relación con cuestiones como ideología y poder. Una de estas líneas parte del presupuesto de considerar la arquitectura como la materialización de ciertas características del cuerpo humano (Hall 1966; Eco 1968; Grahame 1995, 1997). Así, por ejemplo, una pared posee elementos que reproducen, de forma estática, elementos y posturas propias del cuerpo humano, generamos así lo que se ha denominado una "arquitectura del cuerpo" (Eco 1968; Grahame 1995).

Un caso para ilustrar esta propuesta puede ser pensada si imaginamos un grupo de personas interactuando el espacio. La posición del cuerpo define el lugar que cada una de ellas ocupa en la acción, clasificándolas y otorgándole funciones diferentes -por ejemplo, participantes u observadores. Esta práctica social se complementa con gestos, tonos de voz, etc. Así, si partimos del presupuesto de Grahame (1995) de que la región frontal corresponde al espacio público, al encuentro social y el posterior representa el espacio de la no-interacción (Grahame 1995; Tilley 2004), podemos asumir que las personas que están frente a frente están interactuando de forma consensual, mientras que a las que se les da la espalda, están fuera y no participan. Sin embargo, la arquitectura del cuerpo, tiene un carácter extremadamente dinámico y cambiante, ya que un simple movimiento del cuerpo genera transformaciones. Volviendo a nuestro ejemplo, esto posibilitaría que una persona que estaba excluida intencionalmente de la acción pueda entrar a través de un reposicionamiento en el espacio.

Ahora bien, si en lugar de darle la espalda levantamos un muro que deje fuera de la acción a aquellas personas con las que no queremos interactuar, y dentro a los que sí, 
estamos produciendo una herramienta que materializa de forma mucho más efectiva y duradera una intención que antes era apenas generada por el cuerpo. La arquitectura garantiza así la posibilidad de mantener inmutables ciertas relaciones sociales a través de la manipulación del espacio (King 1980; Lefevre 1991; Markus 1993). De esta forma, la organización del espacio efectuada a partir de estructuras arquitectónicas afecta -de la misma manera que lo hace el cuerpo- el tipo de relación entre las personas, y entre las personas y los objetos (Zarankin 2002).

De esta forma ¿por qué no pensar un muro como la representación de una expresión corporal congelada?, un tipo de discurso de las personas residentes hacia los demás (Zarankin 2012). Estos cuerpos congelados, nos expresan la intención de sus habitantes de darnos la espalda, de excluirnos, o-al contrario- estar de frente e incluirnos. Los muros no solo son elementos cotidianos dentro de los cuales nos movemos o que testimonian nuestras vidas, sino que en gran medida la estructuran. Cuerpos de cemento que regulan cuerpos de carne, su materialidad genera los límites y los discursos por donde se desplazan nuestros cuerpos.

Para intentar explicar mejor esta Arquitectura del cuerpo, utilizaremos como ejemplo un estudio realizado en el barrio de la Pampulha en Belo Horizonte ${ }^{5}$, donde uno de los autores vive actualmente (Zarankin 2012). El objetivo fue desarrollar un análisis hermenéutico, de los muros perimetrales de las casas, partiendo del principio que la arquitectura funciona también como un tipo de comunicación no-verbal (Fletcher 1989; Monks 1992; Markus 1993; King 1980; entre otros).

Tomando en consideración estas 3 variables principales: temporalidad, clase social y morfología de la pared, fueron relevados y documentados los diversos tipos de muros existentes en el barrio, entre los que se observó una gran heterogeneidad (por ejemplo, muros o

\footnotetext{
5 La ciudad de Belo Horizonte es la capital del estado brasileño de Minas Gerais desde el año 1897, substituyendo a la ciudad colonial de Ouro Preto. Fue proyectada por el ingeniero Aarão Reis entre los años 1894 y 1897, y fue la primera ciudad moderna brasileña planificada (se tomó como ejemplo para su construcción la ciudad argentina de La Plata). Actualmente, es la tercera ciudad más grande del país, después de São Paulo y Río de Janeiro. En los años 1940 se creó el barrio de Pampulha, junto a un lago artificial, y un parque en el que se construyó la Iglesia de San Francisco de Asís (uno de los primeros proyectos del arquitecto Oscar Niemeyer). Pampulha fue creada por pedido del entonces alcalde de la ciudad (y posteriormente presidente de Brasil), Juscelino Kubitschek (1902-1976). El objetivo era crear un lugar lujoso que contase con todos los encantos y comodidades deseadas por la élite. Según Niemeyer, Pampulha fue un ejemplo de la arquitectura moderna. Asimismo, contó con una serie de rasgos propios que se transformarían en una marca registrada brasilera. Se trata de modelos más leves, con curvas y espacios abiertos acordes con el clima y la geografía del país.
}

paredes de concreto o ladrillo, con o sin decoraciones, rectos o inclinados, altos o bajos, cubiertos por vegetación, pintados, etc.). Es importante reforzar que la elección de los muros perimetrales de las viviendas, como objeto de estudio, no es casual. Son los límites entre los habitantes y las personas extrañas, es decir entre el mundo privado y el público, y por lo tanto se transforman en un elemento de mediación entre ambos grupos. Por ejemplo, una pared alta con alambre de púas, puede ser leída como un discurso de exclusión "de las otras personas", mientras que un grafiti efectuado por alguien extraño en el mismo muro, una crítica a ese rechazo o un intento de domesticar esa pared -de transformarla en un elemento familiar y sobre el cual ejercer algún poder.

Los resultados obtenidos muestran que las casas más antiguas, de 1950 y 1960, pertenecientes a familias acomodadas de la sociedad Belohorizontina, eran construidas con muros perimetrales bajos 0,80 a $1,20 \mathrm{~m}$ de altura, lo que permitía la interacción entre los habitantes de la residencia y los vecinos o transeúntes (Fig. 1). Una lectura de estos parámetros permite observar que el muro actúa apenas como un límite simbólico para delimitar lo privado de lo público. Al mismo tiempo, quienes residen en la casa ven a las demás personas como iguales. En otras palabras, estamos ante un cuerpo que hace frente a la interacción con las otras personas.

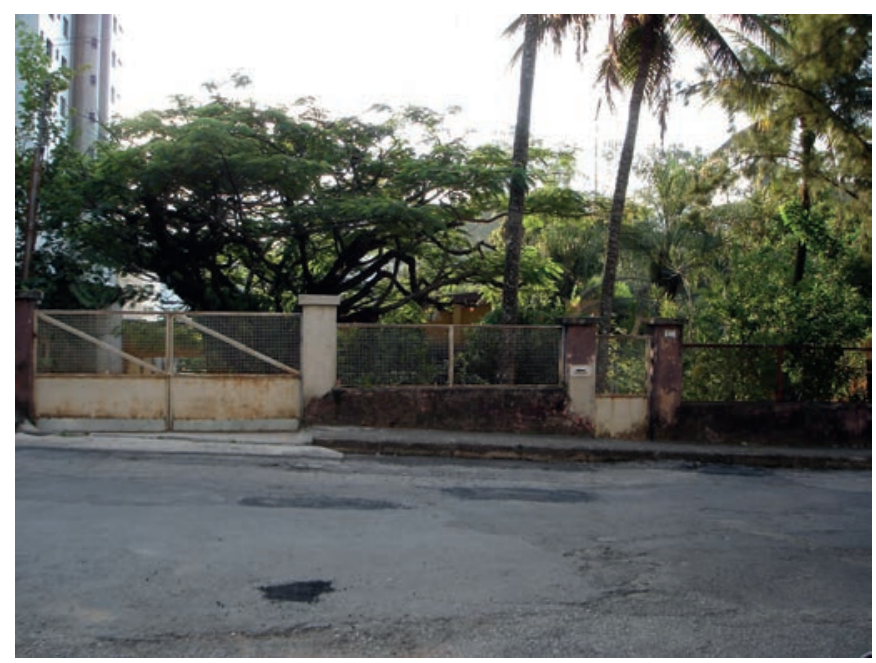

Figura 1. Vista de muro perimetral de una casa de la década de 1960. Fotografía Zarankin 2006.

A mediados de la década de 1970, las mismas casas de la elite, tienen muros cada vez más altos hasta llegar en algunos casos a 6 u 8 metros de altura. Es evidente que los mismos hacen imposible cualquier tipo de relación entre quienes están dentro y quienes están fuera, lo que implicaría ahora un cambio "postural", y un cuerpo que termina 
dándole la espalda a las "otras personas". También es posible observar una mayor heterogeneidad morfológica de estas paredes. Por ejemplo, algunas son cubiertas por plantas o se emplean rocas como materia prima, para generar discursos que "naturalizan" estas nuevas distancias sociales (Fig. 2). Otras veces, al contrario, se utilizan alambres de púas, vidrio molido, rejas con formas de lanza o inclusive cercas eléctricas para reforzar esta separación de las otras personas, que ahora pasan a ser vistas como enemigas (Fig. 3). O sea, no solo se le da la espalda al resto, sino que, de forma extremadamente violenta, le estamos diciendo que quien intente aproximarse podrá ser muerto.
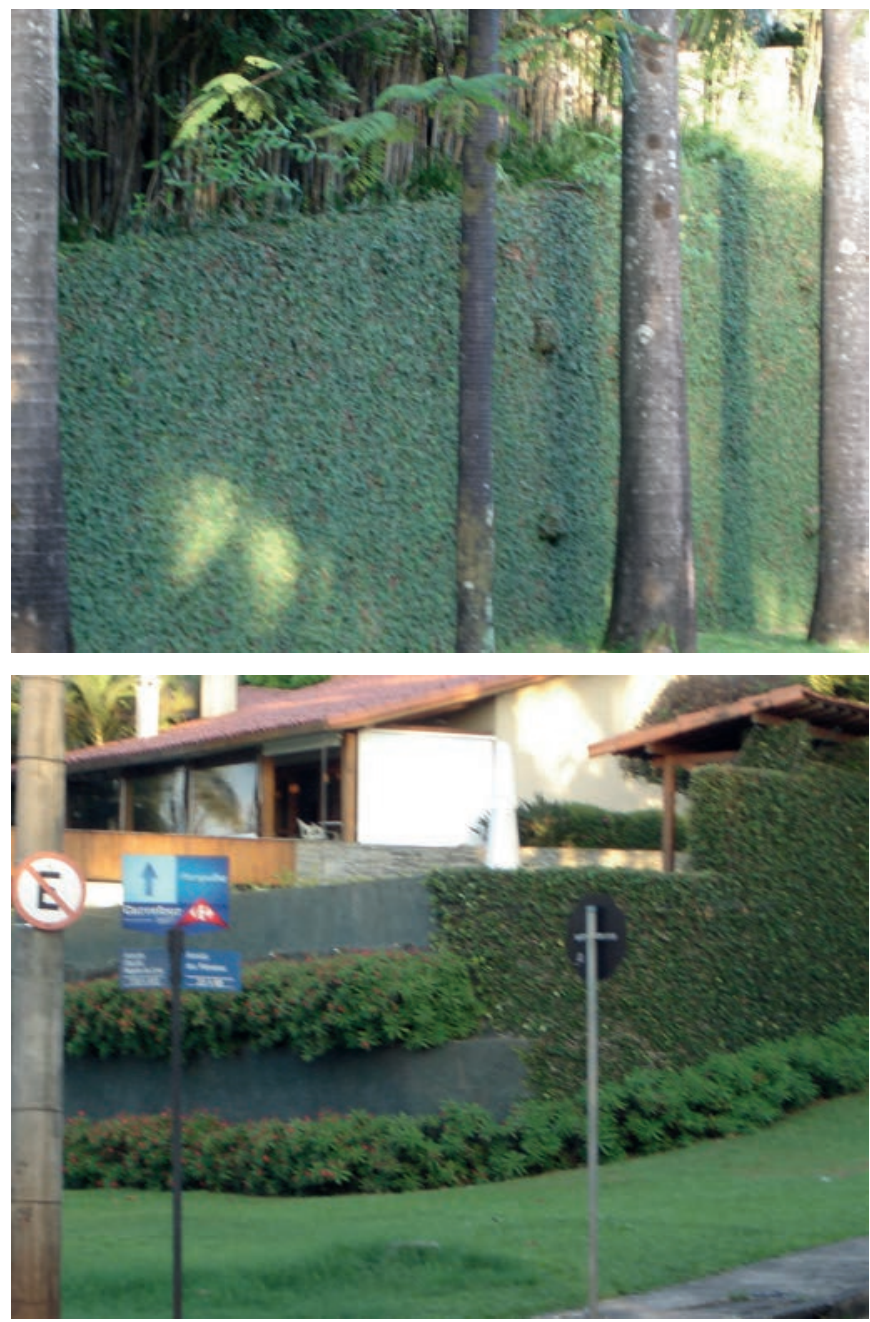

Figura 2. Vista de muros perimetrales que naturalizan su propia existencia. Fotografía Zarankin 2006.

A diferencia de las clases altas, los sectores populares del barrio, también tuvieron un aumento en el tamaño de sus muros perimetrales a través del tiempo. Sin embargo, difícilmente estos ultrapasen los 2 metros, a pesar de que esta altura es suficiente para no permitir
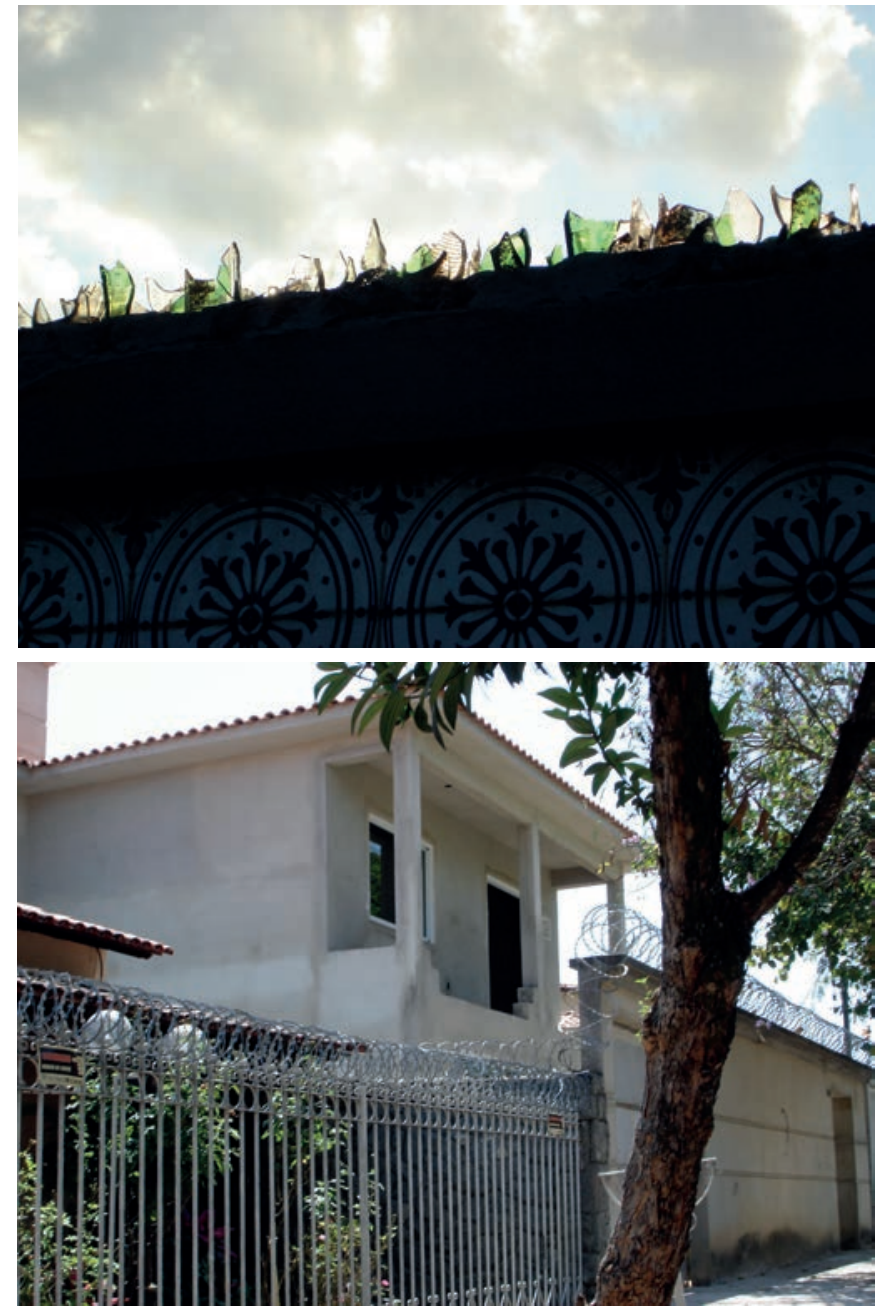

Figura 3. Vista de muros perimetrales que exhiben discursos de violencia. Fotografía Zarankin 2006.

el contacto con los de fuera. Como para estos grupos la interacción social directa es importante en su cotidianeidad, han generado lo que llamamos "pequeños apéndices de sociabilidad" (Zarankin 2010). Esto es, instalar bancos de material, o troncos o sillas viejas en las puertas de las casas (muchas veces hasta con macetas y otros adornos), donde suelen instalarse cuando vuelven de trabajar a fin de tarde o durante la mayor parte del fin de semana, para poder interactuar con las demás personas (Fig. 4).

De forma general, los resultados obtenidos nos permiten reflexionar sobre una nueva conformación del espacio moderno en la sociedad de control (Deleuze 1990), cada vez más lleno de límites y barreras, que implican en cierta medida, el fin de un modo tradicional de vida en sociedad, a través del contacto directo entre las personas. Surgen entonces, nuevos tipos de sociabilidades (y de sociedades), que clasifican y separan a las personas según parámetros diversos (clase social, religión, 

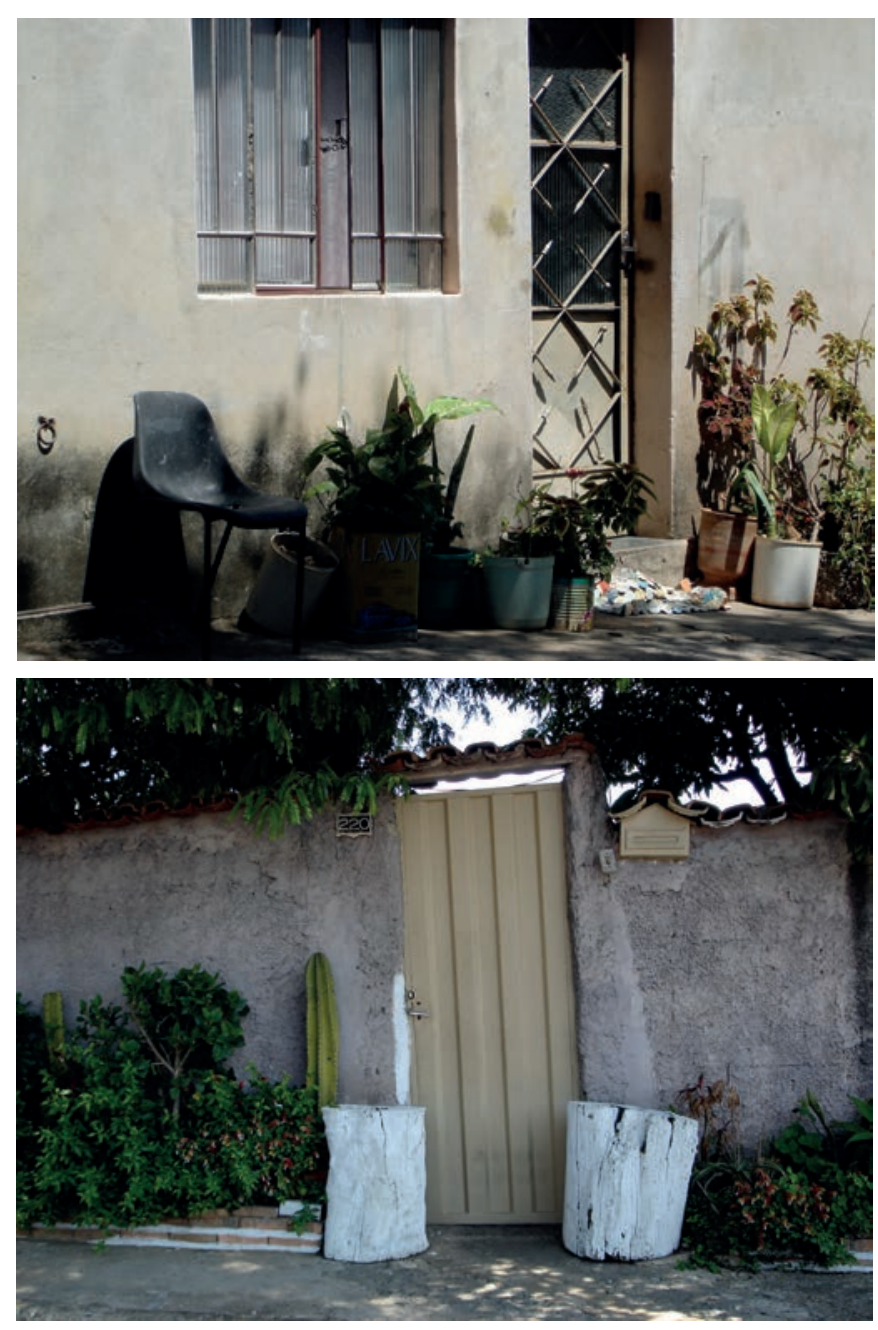

Figura 4. Vista de muros perimetrales de casas de grupos proletarios en los que se observan "pequeños apéndices de sociabilidad". Fotografía Zarankin 2006.

género, edad, etc.). Es en este contexto que los muros paredes refuerzan las categorías ellas-ellos/nosotrasnosotros. Al mismo tiempo, estos "cuerpos congelados", distribuidos por todas partes, terminan dando la espalda a las personas que no tienen, las transforman en "otras personas", volviéndolas invisibles.

En este contexto, queda claro que incorporar los principios estructurantes que organizan y sustentan la sociedad son prioridad para el sistema.

\section{APRENDIENDO A (NO)PENSAR Y ACTUAR; CASAS Y ESCUELAS}

El concepto de habitus primario, también conocido como capital cultural desarrollado por el sociólogo francés Pierre Bourdieu (1977) está relacionado con la capacidad de una determinada estructura social de ser incorporada por los agentes a través de disposiciones para su forma de ser: sentir, pensar, actuar. En otras palabras, Habitus es un sistema de disposiciones incorporadas, que organizan las formas en que las personas perciben el mundo social que les rodea y reaccionan ante él. Para Bourdieu, casas y escuelas son los lugares centrales donde este proceso de incorporación y modelaje de los individuos ocurre.

Por su parte Foucault (1976) sostiene que la arquitectura es un medio eficaz de control y disciplina social (tecnología del poder). A partir de estas bases desde finales del siglo XVIII el sistema viene generando diversas estrategias para controlar el espacio de vida de las personas, a través de codificaciones constructivas la multiplicación de cursos de arquitectura e ingeniería, los colegios y matrículas profesionales, entre otros. El resultado es una homogeneidad constructiva cuyas bases ideológicas sustentan principios de desigualdad, al mismo tiempo que las naturaliza y las vuelve incuestionables. Es dentro de este contexto que adquieren una relevancia particular las estructuras arquitectónicas asociadas a estos procesos de socialización como casas y escuelas (Samson 1990; Parker Pearson y Richards 1994).

Un análisis de la materialidad y espacialidad de estas dos "instituciones" domesticadoras, puede permitirnos entender su funcionamiento como dispositivos del poder. En nuestro caso desarrollamos diversos estudios que fueron tomados como ejemplos de casas y escuelas de la Ciudad de Buenos Aires (Funari y Zarankin 2002, 2005, 2010; Zarankin 1999, 2000).

Como ya explicamos, aplicamos el "modelo Gamma" (Hillier y Hanson 1984), y los índices de Blanton (1994), para analizar la organización espacial de estas estructuras. Los resultados obtenidos muestran una serie de transformaciones simultáneas en casas y escuelas a través del tiempo, que asociamos a cambios en el propio sistema capitalista tanto en Argentina como a nivel global. Pensemos que, en el caso de las escuelas públicas primarias, sus edificios apenas fueron pensados a finales del siglo XVIII (antes funcionaban en edificios preexistentes). Es así que las primeras escuelas estuvieron pautadas por cuestiones simbólicas sobre la importancia de la educación, por lo que muchas veces eran construidas siguiendo morfologías de palacios o templos del mundo clásico. Es en el siglo XIX cuando son incorporados los principios panópticos para servir como bases espaciales de un edificio educativo. Este patrón morfológico espacial se reprodujo en el tiempo a través de más de 200 años en los 
que los cambios estilísticos en sus fachadas fueron apenas de tipo estético para dar la sensación de transformaciones estructurales que nunca ocurrieron (Fig. 5).
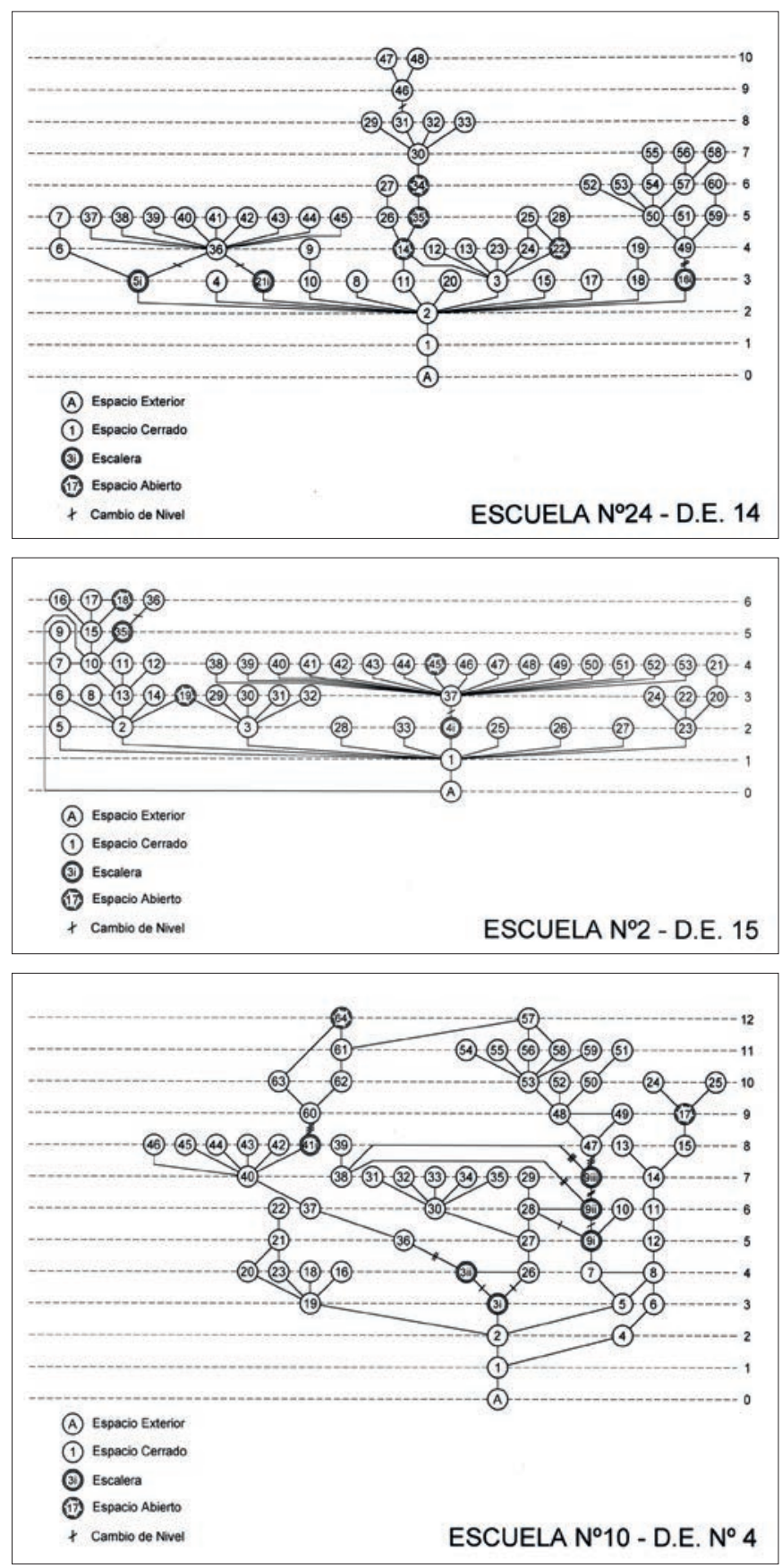

Figura 5. Aplicación de modelo Gama en plantas de escuelas en la que pueden observarse estructuras basadas en un encadenamiento de panópticos. Fuente Zarankin 2001.

Las casas familiares son otro ejemplo interesante de la acción del sistema para construir individuos domesticados. A través del tiempo la tenencia hacia un supuesto mayor confort y flexibilidad enmascara una intensificación en las jerarquías de la estructura, donde las personas residentes son distribuidas espacialmente en función de su posición de poder. En las casas modernas, donde la cantidad de espacios se ha multiplicado de forma geométrica (Zarankin 1999) nadie cuestiona que la "suite master" es ocupada por el dueño o la dueña de la casa, mientras que el segundo mejor cuarto, en caso de una familia con prole lo será por la primogénita o primogénito. Las dependencias en donde se encuentra el personal de servicio dejan claro también el lugar que ocupa en la casa, sirviendo a propietarios y propietarias.

También a través del tiempo en este tipo de estructura doméstica, se observa una restricción en las formas de circular (los cuartos pasan a tener apenas una puerta), y la funcionalidad de los espacios, que antes eran determinadas por la decoración y el mobiliario, en la casa "moderna" pasan a tener destinos predeterminados (cocina, living, dormitorio, escritorio, etc.).

El análisis de la transformación de las casas deja claro cómo el sistema manipula su morfología como forma de generar las bases ideológicas necesarias para construir individuos funcionales al sistema. Personas que por más que se identifiquen como críticos del capitalismo a medida que van abandonando la casa de origen y forman sus propios hogares, este mapa de poder es reproducido sobre los mismos principios de forma inconsciente.

En síntesis, sostenemos que casas y escuelas son dispositivos del poder que funcionan como discursos no-verbales que disciplinan y adoctrinan a sus usuarios para ser funcionales a las necesidades del sistema $(\mathrm{Fu}-$ nari y Zarankin 2002, 2005, 2006, 2010; Zarankin 1999, $2000,2002,2005$ a y 2005b). Es por ello que cambios en las bases estructurales del capitalismo han tenido efectos importantes en la forma en que los espacios son pensados y construidos. Cada fase en la historia del capitalismo tuvo un determinado tipo de casas familiares y escuelas, a partir de las cuales se construye el "individuo normal" que el sistema necesita.

¿Y qué pasa con aquellas personas que en lugar de adaptarse cuestionan el sistema, se revelan y hasta se levantan en contra él?

\section{EL EXTERMINIO DE LO PELIGROSO; CENTROS CLANDESTINOS DE DETENCIÓN (CCD)}

Los espacios y estructuras construidos para segregar, corregir o hasta aniquilar los individuos que se alejan del concepto de "normalidad" es otra temática cada vez más 
frecuente en la Arqueología de la Arquitectura Suramericana. Manicomios, hospitales y prisiones (Lopes 2017; Brandão 2018), están siendo foco de investigaciones realizadas principalmente por jóvenes especialistas en arqueología. Dentro de esta temática uno de los casos de investigaciones que crece con más fuerza son los estudios asociados con las estrategias represivas de las dictaduras militares post 1950 (Fuenzalida 2009; Bianchi 2008; Cáceres 2011; Marín 2014; Cattaneo 2015; Belle 2017; Costa 2017; Maguire y Costa 2018; Lemos Murta 2019; entre otros). Partimos del principio que tanto la desaparición de personas como la utilización de centros clandestinos de detención (CCDs) fueron las metodologías predominantes de estos gobiernos autoritarios para aniquilar la disidencia (Funari y Zarankin 2006). La Arqueología, por sus características específicas de trabajo, viene reconstruyendo "historias no oficiales sobre la represión", y la búsqueda de la verdad (Funari 2019).

Estos trabajos no solo permiten comprender y evidenciar una ingeniería del terror, sino también ayudar a la construcción de una memoria material del genocidio en la región (Zarankin y Salerno 2012). Actualmente el desarrollo de espacios de diálogo entre colegas latinoamericanos ha permitido generar entendimientos regionales, a partir de los cuales se están discutiendo semejanzas y diferencias en los procesos represivos vividos en cada país (Zarankin y Salerno 2011). De todas formas, actualmente el crecimiento de gobiernos de ultraderecha en la región es un factor que coloca en riesgo los avances conseguidos, por lo que hoy más que nunca el reto es garantizar el mantenimiento de los trabajos, potenciando además la producción de nuevas propuestas de investigación.

Si bien los distintos trabajos sobre la temática desde sus orígenes en la década de 1980, tienen múltiples objetivos y objetos de estudios (desde grafitis hasta los restos de personas desaparecidas), uno de los que más interés ha tenido, es el estudio de los espacios de confinamiento de los prisioneros, muchas veces en los denominados centros clandestinos de detención (Zarankin, Salerno y Perosino 2012).

Uno de los casos que hemos trabajado a partir de herramientas de la Arqueología de la Arquitectura, es el CCD argentino conocido como "Club Atlético" (sobrenombre derivado de las iniciales del verdadero nombre del lugar - "Centro Antisubversivo"). En ese escenario desarrollamos en 2003 la propuesta "Arqueología como memoria: Intervenciones arqueológicas en el centro clandestino de detención y tortura Club Atlético" (Bianchi Villelli y Zarankin 2002).
Propusimos 2 objetivos principales para el proyecto, por un lado, comprender la planificación espacial y material del CCD como instrumento represivo y por el otro contribuir a la construcción de un espacio de la memoria, que funcionase como representación material sobre los horrores que ocurrieron en ese lugar. Los trabajos fueron desarrollados por un colectivo multidisciplinario que contaba, además de profesionales de la arqueología, con otras disciplinas como arquitectura, antropología, historia y conservación, coordinados por una comisión que incluía sobrevivientes del CCD o familiares de víctimas, además de integrantes de organizaciones de derechos humanos y del gobierno de la ciudad.

Los análisis efectuados sobre la arquitectura y organización espacial partieron de un plano producido por las propias personas sobrevivientes del campo, a partir de las cuales aplicamos los modelos Gamma de Hillier y Hanson (1984) y los índices de Blanton (1994) que ya comentamos antes. Los resultados obtenidos muestran como elemento organizativo del espacio un parámetro de maximización y operatividad de los procedimientos represivos en el que su corazón/centro, es la sala de tortura, que representa la materialización del sadismo con la que fue proyectado el CCD (Fig. 6).

Por otra parte, el espacio del "Club Atlético" fue dividido en 2 ejes. Uno superior (completamente destruido cuando se demolió el lugar) donde funcionaba la parte burocrática del CCD. Existe también un subsuelo (que sí sobrevivió) que acoge a las personas prisioneras y donde se ubican las salas de tortura. Esta organización divide y clasifica a las personas dentro del mismo, delimitando espacios de circulación y permanencia de quienes padecieron la detención.

Los CCDs representan una parte central de toda una ingeniería de la destrucción (Zarankin y Salerno 2012, pensada para maximizar el control, la sumisión, la tortura y la eliminación de las y los detenidos. Su funcionamiento y organización espacial se basa en criterios funcionales y simbólicos que maximizan sus efectos. Por ejemplo, la ubicación de las salas de tortura en la zona central minimiza el traslado de las personas detenidas en el espacio, a la vez que los gritos de quienes sufrían tortura están siendo escuchadas por quienes en ese momento ocupan las celdas, generando una situación de suplicio permanente. De la misma forma, las sensaciones de la gente detenida son más intensas a medida que avanzan hacia el interior del CCD. Allí es donde el mayor nivel de aislamiento contrasta con una diversidad de estímulos sensoriales "negativos", entre otros, térmicos (calor intenso en el verano y frío en el invierno), olores de 


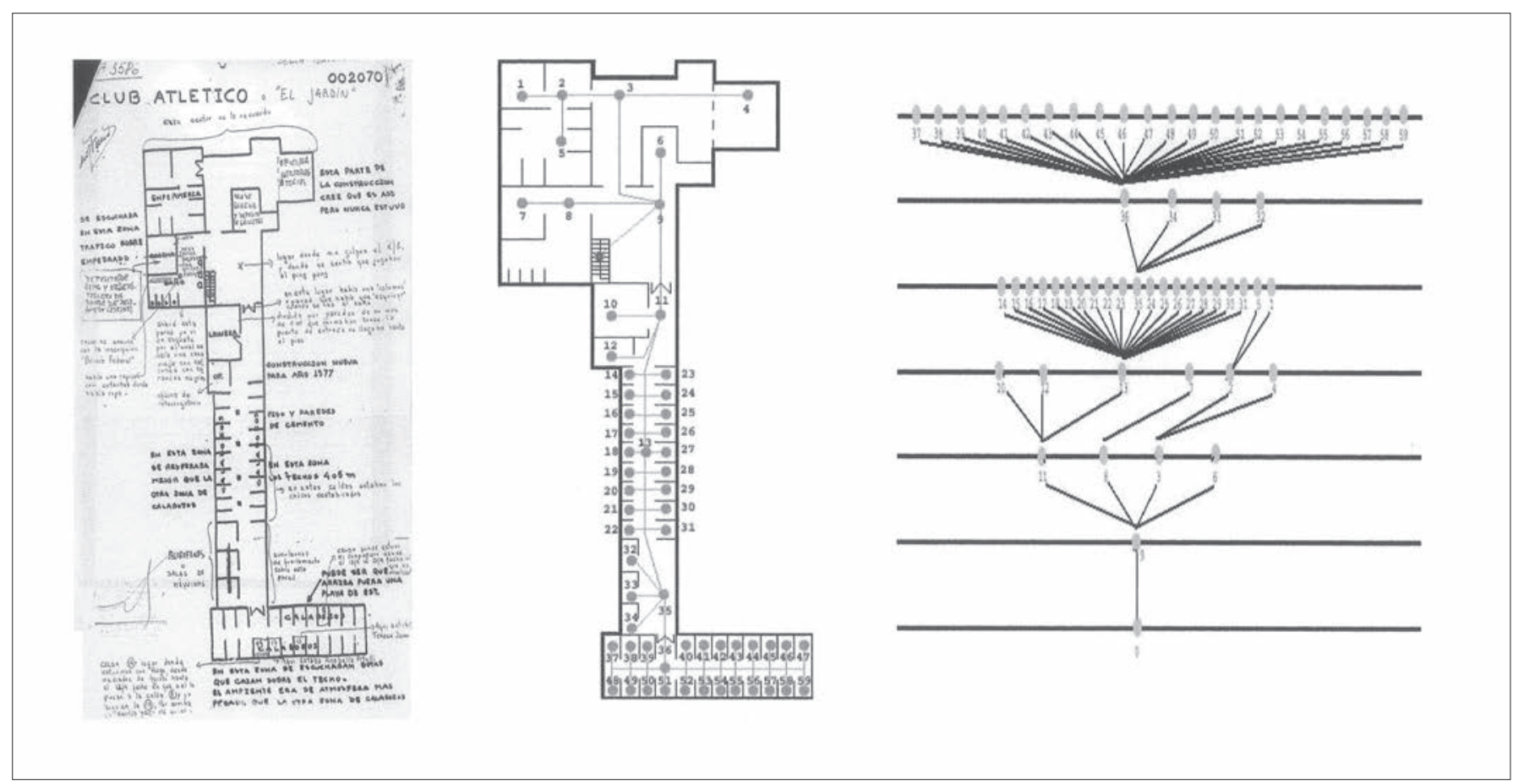

Figura 6. Plano del subsuelo del Club Atlético dibujado por los supervivientes de acuerdo a sus recuerdos (Benítez, Enríquez y Di Ciano 2001: 10) y aplicación del modelo Gamma. Fuente: Zarankin 2006.

cuerpos y fluidos humanos, humedad, falta de ventilación, que se ven reforzados por la dureza de las paredes y el piso -donde se ubicaban-, sumado a los gritos y llantos de las otras personas detenidas. Recordemos que estas tenían siempre vendas o capuchas, por lo que estamos ante una estructura para ser percibida de maneras alternativas a la visión, a través de sentidos como el tacto, el sonido o el olor. Un "no-lugar" que produce "no-personas" (Zarankin y Niro 2006).

\section{PALABRAS FINALES; ARQUITECTURA, COTIDIANEIDAD Y OPRESIÓN}

Hace tiempo vienen creciendo dentro de las arqueologías suramericanas, movimientos "decoloniales" que buscan crear una agenda propia, acorde a su historia y contextos socio-políticos. A partir de posiciones que entienden la Arqueología como una forma de acción política (McGuire 2008), se busca repensar de manera crítica los procesos de reproducción de los sistemas de poder en nuestro cotidiano, así como sus múltiples conexiones en el ámbito regional. En este contexto, la Arqueología de la Arquitectura puede funcionar como una herramienta relevante, al servicio de la construcción de mecanismos de denuncia y cuestionamiento social.
En nuestro caso, a través de reunir y resumir algunos casos de estudio que realizamos en los últimos 20 años, intentamos mostrar que la arquitectura es mucho más que apenas un elemento técnico destinado a suplir necesidades humanas, como refugio y protección. Al contrario, la arquitectura entendida no solo como la materialización de una ideología y como una tecnología del poder, nos abre todo un universo de investigación para entender las estrategias de reproducción del sistema.

El biopoder actúa sobre los individuos en diversos niveles a través de una "explosión" de variadas y numerosas técnicas, para lograr la subyugación de los cuerpos y el control de las poblaciones alrededor de un modelo de lo que es considerado "normal" (Foucault 1980). Casas y las escuelas, son sin duda dispositivos centrales para modelar los individuos deseados ${ }^{6}$ por el sistema, mientras que las paredes y muros establecen las formas y ritmos de este proceso de construcción disciplinar. Para quienes se revelan, o quienes se alejen de los parámetros considerados normales, existen las instituciones totales. Las "correctivas" (hospitales, prisiones, manicomios, etc.) para los casos considerados "recuperables" y las "exterminadoras" (como los Centros Clandestinos

\footnotetext{
${ }^{6}$ Aquellos que de manera acrítica cumplan con su función y cuyo accionar ayude a mantener y reproducir las relaciones de poder.
} 
de Detención) para las situaciones en las que el sistema directamente quiere hacer desaparecer las "anomalías".

Otra cuestión que nuestro trabajo permite observar, analizando las transformaciones en la arquitectura, son los cambios en el propio biopoder, principalmente con el paso de la sociedad disciplinaria a la de control. Según Foucault, el dispositivo disciplinario ejercido sobre los cuerpos de los individuos: personas trabajadoras, prisioneras, pacientes, estudiantes, tuvo lugar históricamente en la sociedad industrial que alcanzó su apogeo con la organización científica del trabajo. La vida humana, en ese momento, se transformó en producción, y la sociedad disciplinaria podría entonces considerarse una "máquina para producir" personas trabajadoras. Con la decadencia de la era fabril, entramos en el tiempo de la sociedad de control (Deleuze 1990), en el que el poder, que antes estaba centrado, en el "interior" de las fábricas, hospitales, cuarteles, escuelas, se extiende, incluyendo nuevos elementos de control permanentes, modulares y difusos. Casas y escuelas, espacios de confinamiento y muros, y sus transformaciones a través del tiempo, son ejemplos a partir de los cuales podemos reflexionar sobre las consecuencias de estos cambios y sus efectos sociales y materiales.

En síntesis, los casos presentados buscan entender las lógicas a través del tiempo a partir de la cual se levantan límites y barreras que dividen y clasifican personas, objetos y lugares. Sin duda una aproximación arqueológica de las formas y discursos de estos "cuerpos congelados" puede ser importantes para la búsqueda de brechas y espacios de resistencia que estimulen una transformación social hacia organizaciones más plurales, democráticas y con menos muros.

\section{AGRADECIMIENTOS}

A Agustín Azkarate por su invitación a participar de este monográfico sobre Arqueología de la Arquitectura. Finalmente, al Departamento de Antropología y Arqueología de la UFMG y a la UNICAMP.

\section{BIBLIOGRAFÍA}

Andrade Lima, T. 1999: "El huevo de la serpiente: Una arqueología del capitalismo embrionario en el Rio de Janeiro del siglo XIX", en F. Acuto y A. Zarankin (eds.), Sed Non Satiata: teoría social en la arqueología latinoamericana contemporánea, pp. 189-238. Buenos Aires, Ediciones Del Tridente.
Ayán, X. 2003: "Arquitectura como tecnología de construcción de la realidad social", Arqueología de la Arquitectura, 2, pp. 17-24.

Azkarate, A. 2001: Arqueología de la Arquitectura (Experiencias de investigación desde la UPV/EHU). Lección inaugural del curso académico 2001-2002. Ikasturte hasiera emateko hitzaldia. Universidad del País Vasco (UPV/EHU).

Azkarate, A. 2013: "La construcción y lo construido. Arqueología de la Arquitectura", en J. A. Quirós (dir), La Materialidad de la historia. La arqueología en los inicios del siglo XXI, pp. 271-298. Madrid, Akal.

Bachelard, G. 1975: La Poética del Espacio. México, Fondo de Cultura.

Bellé, F. M. 2017: Memoria em Conflito: Dilemas da arqueologia da repressão em Porto Alegre/RS. Defesa de Mestrado. Disponible en: http://www.fafich.ufmg.br/ppgan/wp-content/uploads/2017/08/MatheusFuscaldo-Belle-31-agosto-2017-1.pdf (visitado en septiembre 2017).

Benítez, M.; Enríquez, Z. y Di Ciano, L. 2001: "El Club Atletico", Revista de la Asociación de ex detenidos desaparecidos, 1 (2), pp. 1-18.

Bezerra, M. 2013: "Os sentidos contemporâneos das coisas do passado: reflexões a partir da Amazônia”, Revista de Arqueologia Pública, 7 (2).

Bianchi, S. 2008: El Pozo (ex servicio de informaciones). Un centro clandestino de detención, desaparición, tortura y muerte de personas de la ciudad de Rosario, Argentina. Antropología politica del pasado reciente. Rosario, Argentina, Ediciones Prohistoria.

Bianchi Villelli, M. y Zarankin, A. 2002: Arqueología como memoria. Intervenciones arqueológicas Intervenciones arqueológicas en el Centro Clandestino de Detención y Tortura “Club Atlético”. Plan de Trabajo aceptado por la Comisión de Trabajo y Consenso del Proyecto "Recuperación de la memoria del Centro Clandestino de Detención y Tortura Club Atlético". Dirección General de Derechos Humanos. Gobierno de la Ciudad Autónoma de Buenos Aires.

Blanton, R. 1994: Houses end Households. New York, Plenum Press.

Bourdieu, P. 1977: Outline of a Theory of Practice. Cambridge, Cambridge University Press.

Brandão, J. 2018: "Marieta e Josefa no prédio da loucura: uma arqueologia dos espaços manicomiais", Revista de Arqueologia - Dossiê Arqueologia da Infância, 31 (2).

Buchli V. y Lucas, G. 2001: Archaeologies of the Contemporary Past. London, Routledge.

Cáceres, I. 2011: Detenidos Desaparecidos en Chile. Arqueología de la Muerte Negada. Tesis para optar al Título de Arqueólogo. Facultad de Ciencias Sociales, Departamento de Antropología, Universidad de Chile, Santiago.

Cattaneo, C. 2015: "Memorias de la represión en los muros. La base militar argentina de Santa Lucía. Depto. De Monteros, Tucumán (1975-1982)”, La Zaranda de Ideas, Revista de Jóvenes Investigadores en Arqueología, 13 (2), pp. 91-106.

Certeau, M. de. 1980: La Invención de lo Cotidiano; 1 Artes de Hacer. México, Universidad Iberoamericana.

Conway, H. y Roenisch, R. 1994: Understanding Architecture. Londres, Routledge.

Costa, D. 2017: O passado que ninguém quer lembrar: uma arqueologia dos espaços de repressão em Belo Horizonte. Trabalho de conclusão de curso. Universidade Federal de Minas Gerais, Faculdade de Filosofia e Ciências Humanas.

Deleuze, G. 1990: Controle e devenir. Conversacoes 1972-1990. São Paulo, Editora 34.

Eco, U. 1968: La Estructura Ausente. Barcelona, Lumen.

Fletcher, R. 1989: "The Messages of Material Behavior: a Preliminary Discussion of Non-verbal Meaning", en I. Hodder (ed.), The Meaning of the Things: Material Culture and Symbolic Expression, pp. 33-39. Londres, Harper Collings.

Foucault, M. 1976: Vigilar y Castigar. El Nacimiento de la Prisión. México, Siglo XXI.

Foucault, M. 1980: Power/Knowledge: Selected Interviews and Other Writings, 1972-1977. New York, Phanteon Book.

Fuenzalida, N. 2009: Villa Grimaldi, Análisis de la Configuración del Espacio en relación a las Estrategias de Represión y Control de los Cuerpos de Detenidos y Torturados. Hacia una Arqueología de la Represión Política 
en Chile. Informe de Práctica Profesional. Departamento de Antropología, Universidad de Chile.

Funari, P. 1995: "A cultura material e a construção da mitologia Bandeirante: Problemas da identidade nacional brasileira”, Ideais 1, pp. 29-48.

Funari, P. 1997: "Archaeology, History and Historical Archaeology in South America", International Journal of Historical Archaeology, 1, pp. 137148.

Funari, P. 1999: "Historical Archaeology from a World Perspective", en P. Funari, M. Hall y S. Jones (eds.), Historical Archaeology: Back from the Edge, pp. 37-66. Londres, Routledge.

Funari, P. 2019: Arqueologia e direitos humanos, uma introdução. Curitiba: Appris.

Funari, P. y Zarankin, A. 2002: “A social archaeology of housing from a Latin American perspective: a case study", Journal of Social Archaeology, 3 (1), pp. 23-45.

Funari, P. y Zarankin, A. 2004: Arqueologia Historica en America del Sur; Los desafios del siglo XXI. Bogotá, Ediciones Uniandes.

Funari, P. y Zarankin, A. 2005: "Cultura material escolar: o papel da arquitetura”, Pró-Posições, 16 (1), pp. 135-144.

Funari, P. y Zarankin, A. (orgs.) 2006: Arqueología de la Represión y la Resistencia en América Latina (1960-1980). Córdoba: Encuentro.

Funari, P. y Zarankin, A. 2010: "Pompeia; cidade, quotidiano e poder a luz das discussoes epistemologicas recentes", en A. Campos, G. Silva, M. Nader, S. Franco y S. Feldman (orgs.), A Cidade a prova do Tempo; vida cotidiana e relacões de poder em amientes urbanos, pp. 7-27. Vitoria, GM Editora/Université de Paris-Est.

Giddens, A. 1979: Central Problems in Social Theory: Action Structure and Contradiction in Social Analysis. Londres, Macmillan.

Giddens, A. 1990: La Constitución del Sociedad. Bases para la teória de la estructuración. Buenos Aires, Amorrortu Editores.

Glassie, H. 1975: Folk Housing in Middle Virginia. Knoxville, University of Tennessee Press.

Gnecco, C. y Ayala, P. (eds.) 2010: Pueblos Indigenas y Arqueología en América Latina. Bogota, Ediciones Uniandes.

Grahame, M. 1995: The House of Pompeii: Space and Social Interaction. Tesis de doctorado, Faculty of Arts, Departament of Archaeology, Southampton University, Gran Bretaña.

Grahame, M. 1997: "Public and private in the Roman house: the spatial order of the Casa del Fauno", Journal of Roman Archaeology, supplement 22, pp. 137-164.

Gottdiener, M. 1985: A Producão Social do Espaco Urbano. São Paulo, Edusp.

Gottdiener, M. 1995: Postmodernism Semiotics: Material Culture and Forms of Postmodern Life. Oxford, Blackwell.

Haber, A. 2011: "Nometodología Payanesa: Notas de metodología indisciplinada", Revista Chilena de Antropologia, 23: pp. 9-41.

Hage, P. 1979: "Graph Theory as Estructural Model in Cultural Anthropology", Annual Review of Anthropolgy, 8: pp. 115-136.

Hall, E. 1966: A Dimensao Oculta. Rio de Janeiro, Francisco Alves S.A.

Hillier, B. y Hanson, J. 1984: The Social Logic of Space. Cambridge, Cambridge University Press.

Hodder, I. 1982: Simbols in Action. Londres, Cambridge University Press.

Hodder, I. 1994: "Architecture and Meaning: The Example of Neolithic Houses and Tumbs. Architecture and Order. Aproaches to Social Space", en M. Parker Pearson y C. Richard (eds.), Architecture and Order. Approaches to Social Space, pp. 73-86. Londres, Routledge.

Hodder, I., Shanks, M., Alexandri, A., Buchli, V., Carman, J., Last, J. y Lucas, G. 1995: Interpreting Archaeology; Finding meaning in the past. Londres, Routledge.

Johnson, M. 1996: An archaeology of capitalism. Oxford, Blackwell.

Kent, S. 1990: Domestic Architecture and the Use of Space. New Direction in Archaeology. Cambridge, Cambridge University Press.

King, A. (ed.) 1980: Buildings and Society; Essays on the Social Development of the Built Environment. Boston, Routledge-Keagan Paul.

Knights, C. 1994: "The Spatiality of the Roman Domestic Setting: An Interpretation of Simbolic Content”, en M. Parker Pearson y C. Richards (eds.),
Architecture and Order. Aproaches to Social Space. Londres, Routledge, pp. 113-146.

Lefebvre, H. 1991: The Production of Space. Oxford, Blackwell.

Lemos Murta, C. 2019: Arquitetando o terror: um estudo sensorial dos centros de detenção oficiais e clandestinos da ditadura civil-militar do Brasil (1964-1985). Tese de Doutorado: Universidade Federal de Sergipe, Programa de Pós-graduação em Arqueologia.

Leone, M. 1977: "The New Mormon Temple in Washington DC", en L. Ferguson (ed.), Historical Archaeology and the Importance of Material Things, pp. 43-61. Arizona, Society for Historical Archaeology. Special Publication Series (2).

Lopes Rhuan, C. S. 2017: Tempos, espaços e cultura material na Vila Santo Antônio do Prata, Pará - Arqueologia em uma Instituição Total Amazônica. Tese Doutorado, Universidade Federal do Pará, Belém.

Maguire, P. y Costa, D. 2018: "Scientific torture? Scientism and the marks of torture inside a police station in Belo Horizonte, Brazil". Vibrant, virtual Braz Anthr., 15 (3).

Mañana Borrazás, P., Blanco Rotea, R. y Ayán Vila, X. 2002: Arqueotectura 1: bases teórico metodológicas para una arqueología de la arquitectura. Santiago de Compostela, Laboratorio de Patrimonio, Paleoambiente e Paisaxe, Instituto de Investigacións Tecnolóxicas, Universidade de Santiago de Compostela.

Marín, C. 2014: “Arqueología de los campos de concentración del s. XX: Argentina, Chile, Uruguay y España”, Arkeogazte, Euskal Herria 4, pp. 159-182.

Markus, T. 1993: Buildings and Power; Freedom and Control in the Origin of Modern Buildings Types. Oxford, Blackwell.

McGuire, R. H. 1991: "Building Power in Cultural Landscape of Broome County, New York 1880 to 1940 ”, en R. H. McGuire y R. Paynter (eds.), The Archaeology of Inequality, pp. 102-124. Massachusetts, Blackwell.

McGuire, R. H. 2008: Archaeology as Political Action. Londres, University of California Press.

Miller, D. 1987: Material Culture and Mass Consuption. Oxford, Blackwell.

Monks, G. 1992: "Architectual Simbolism and Non-verbal communication at Upp.er Fort Garry”, Historical Archaeology, 26 (2), pp. 37-57.

Nielsen, A. 1995: "Architectural Perfonmance and Social Reprduction of Power. Expanding Archaeology", en J. Skibo, W. Walker y A. Nilsen, (eds.), Expanding Archaeology (Foundations of Archaeological Inquiry), pp. 47-66 Utah, University of Utah Press.

Nuttgens, P. 1983: Historia de la Arquitectura. Madrid, Ediciones Destino.

Orser Jr., Ch. 1996: A Historical Archaeology of the Modern World. New York, Plenum Press.

Orser Jr., Ch. y Funari, P. 2001: "Archaeology and Slave Resistance and Rebellion”, World Archaeology, 33, pp. 61-72.

Panofsky, E. 1957: Arquitectura Gótica y Pensamiento Escolástico. Madrid, Ed. La Piqueta.

Parker Pearson, M. y Richards, C. (eds.) 1994: Architecture and Order. Approaches to Social Space. Londres, Routledge.

Pellini, J. 2016: Arqueologia e os Sentidos: Entrando na toca do coelho. Curitiba, Editora Prismas.

Ruibal, A. G. 2014: "Returning to Where We Have Never Been: Excavating the Ruins of Modernity", en B. Olsen y P. Pétursdóttir (eds.), Ruin Memories Materialities, Aesthetics and the Archaeology of the Recent Past, pp. 367-389. London, Routledge.

Salerno, M. 2006: Arqueología de la Indumentaria. Prácticas e Identidad en los Confins del Mundo Moderno (Antártida, siglo XIX). Buenos Aires, Del Tridente.

Samson, R. (comp.) 1990: The Social Archaeology of Houses. Edinburgh, University Press.

Sennett, R. 1974: O Declinio do Homem Público. São Paulo, Companhia Das Letras.

Shanks, M. y Tilley, C. 1987: Reconstruting Archaeology. Londres, Routledge. Soares, F. C. 2015: Arqueologia das fortificações: perspectivas. Florianópolis, Lagoa Editora.

Steadman, S. 1996; "Recent Research in the Archaeology of Architecture: Beyond the Foundations", Journal of Archaeological Research, 4 (1), pp. 51-93. 
Steadman, S. 2015: Archaeology of Domestic Architecture and the Human Use of Space. London, Routledge.

Symanski, L. C. P. 2001: "Exposição e Isolamento: Práticas de descarte de refugo e mudanças de visão de mundo em um ambiente rural - o sítio Fazenda Camurugi (BA)", Revista de Divulgação Cientifica, 4, pp. 113-137.

Tilley, C. 2004: The Materiality of Stone. Oxford, Berg.

Trigger, B. 1989: A History of Archaeological Thought. Cambridge, Cambridge University Press

Zarankin, A. 1999: "Casa Tomada; sistema, poder y vivienda doméstica", en A. Zarankin y F. Acuto (eds.), Sed Non Satiata; Teoría Social en la Arqueología Latinoamericana Contemporánea, pp. 239-272. Buenos Aires, Del Tridente.

Zarankin, A. 2000: "Arqueología de la arquitectura. Another brick in the wall", Revista do Museu de Arqueologia e Etnologia da Universidade de São Paulo, número especial, pp. 119-128.

Zarankin, A. 2002: Paredes que Domesticam: Arqueologia da Arquitetura Escolar Capitalista; O caso de Buenos Aires. Campinas, Centro de Historia da Arte e Arqueologia (IFCH-UNICAMP).

Zarankin, A. 2005a: "Arqueología de la Arquitectura, modelando al individuo disciplinado en la sociedad capitalista", Revista de Arqueologia Americana, 22 (1), pp. 25-41.

Zarankin, A. 2005b: “"Walls of Domestication»: Archaeology of the architecture of capitalist elementary public schools; The case of Buenos Aires", en P. Funari, A. Zarankin y E. Stovel (orgs.), Global Archaeological Theory: contextual voices and contemporary thoughts, pp. 237-264. New York, Kluwer-Plenum.

Zarankin, A. 2008: "Los Guardianes del Capital; Arqueología de la Arquitectura de los Bancos de la Ciudad de Buenos Aires", en F. Acuto y A. Zarankin (orgs.), Sed Non Satiata II, acercamientos sociales en la arqueología latinoamericana, v. 1, pp. 325-339. Córdoba, Encuentro Grupo Editor.

Zarankin, A. 2012: “Corpos congelados: uma leitura metafórica de paredes e muros em Belo Horizonte, MG", en R. Andrade, J. Macedo y C. Terra (orgs.), Arqueologia na paisagem: novos valores, dilemas e instrumentos, pp. 18-33. Rio de Janeiro, Rio Books.

Zarankin, A. y Niro, C. 2006: "La materialización del sadismo: Arqueología de la arquitectura de los centros clandestinos de detención de la dictadura militar argentina (1976-1983)", en P. Funari y A. Zarankin (comps.), Arqueología de la Represión y Resistencia en América Latina (1960-1980), pp. 159-182. Córdoba, Brujas.

Zarankin, A. y Salerno, M. 2011: "The Engineering of Genocide: An Archaeology of Dictatorship in Argentina", en A. Myers y G. Moshenska (eds.), Archaeologies of Internment, One World Archaeology, pp. 207-227. New York, Springer.

Zarankin, A. y Salerno, M. 2012: "«Todo está guardado en la memoria». Reflexiones sobre los espacios para la memoria de la dictadura en Buenos Aires (Argentina)", en A. Zarankin; M. Salerno y M. Perosino (Orgs.), Historias desaparecidas: arqueología, memoria y violencia política, pp. 143-174. Cordoba, Encuentro.

Zarankin, A., Salerno, M. y Perosino, M. 2012: “Arqueologías de la clandestinidad. Una revisión de los trabajos efectuados en los centros de detención clandestinos de la última dictadura militar en Argentina", Revista Universitaria de Historia Militar, 2, pp. 1-36.

Zarankin, A. y Senatore, M. (orgs.) 2002: Arqueologia da Sociedade Moderna na América do Sul: Cultura Material, Discursos e Práticas. Buenos Aires, Ediciones del Tridente.

Zevi, F. 1969: Arquitectura in Nuce; Una definición de arquitectura. Madrid, Aguilar. 